\title{
El desarrollo del mercado audiovisual en Argentina: una industria de exportación
}

Ethel Pis Diez ${ }^{1}$
Fausto García ${ }^{2}$

Recibido: 2013-10-01

Aprobado por pares: 2014-01-17

Envío a pares: 2013-10-03

Aceptado: 2014-02-26

DOI: 10.5294/pacla.2014.17.4.7

Para citar este artículo / To reference this article / Para citar este artigo

Pis Diez, E. y García, F. Diciembre de 2014. El desarrollo del mercado audiovisual en Argentina: una industria de exportación. Palabra Clave 17 (4), 1137-1167. D0I:10.5294/ pacla.2014.17.4.7

\section{Resumen}

Los formatos de televisión se presentan como un campo de investigación apropiado para comprender el dinamismo creciente de la industria televisiva caracterizado por la interacción constante entre lo local y lo global, lo tradicional y lo innovador. El propósito de este artículo es presentar los resultados de una investigación acerca de los cambios generados por la comercialización internacional de formatos en los procesos de creación, producción y distribución de contenidos en el mercado televisivo argentino. La investigación gira en torno al conocimiento pragmático de los profesionales del sector, y se centra en las estrategias de entrada en el mercado internacional desarrolladas por las empresas audiovisuales argentinas en los últimos veinte años.

\section{Palabras clave}

Televisión, producción televisiva, Argentina, globalización. (Fuente: Tesauro de la Unesco).

\footnotetext{
Profesora Titular de la Facultad de Comunicación, Universidad Austral, Buenos Aires. episdiez@austral.edu.ar

Profesor Asociado del IAE Business School, Universidad Austral, Buenos Aires. fgarcia@iae.edu.ar
} 


\title{
Development of the Audiovisual Market in Argentina: An Export Industry
}

\begin{abstract}
Television formats are presented as an appropriate field of study to understand the growing momentum in the television industry, which is characterized by constant interaction between what is local and global, traditional and innovative. The purpose of this article is to present the results of a study on how international marketing of formats has changed content creation, production and distribution processes in the Argentine television market. The research revolves around the pragmatic knowledge of industry professionals, and focuses on the international market-entry strategies developed by Argentine media companies over the past twenty years.
\end{abstract}

\section{Keywords}

Television, television production, Argentina, globalization. (Source: Unesco Thesaurus). 


\section{0 desenvolvimento do mercado audiovisual na Argentina: uma indústria de exportação}

\section{Resumo}

Os formatos de televisão se apresentam como um campo de pesquisa apropriado para compreender o dinamismo crescente da indústria televisiva caracterizado pela interação constante entre o local e o global, e o tradicional e o inovador. O propósito deste artigo é apresentar os resultados de uma pesquisa sobre as mudanças geradas pela comercialização internacional de formatos nos processos de criação, produção e distribuição de conteúdos no mercado televisivo argentino. A pesquisa gira em torno do conhecimento pragmático dos profissionais do setor e centraliza-se nas estratégias de entrada no mercado internacional desenvolvidas pelas empresas audiovisuais argentinas nos últimos vinte anos.

\section{Palavras-chave}

Televisão, produção televisiva, Argentina, globalização. (Fonte: Tesauro da Unesco). 


\section{Introducción}

La industria de los medios es un sector crecientemente internacional, con productos que tienen una llegada cada vez más amplia. A fines de los años ochenta, y durante los noventa, la competencia y las nuevas tecnologías llevaron a las empresas de medios nacionales a formar parte de corporaciones globales para beneficiarse de las economías de escala y de enfoque. Dentro de las estrategias de diversificación geográfica la exportación de contenidos a otros mercados ha sido percibida por muchas empresas del sector como una de las mejores formas de recuperar la inversión cuando el producto ha sido exitoso.

Una de las estrategias de entrada más exitosas en la industria televisiva a través del contenido ha sido el desarrollo y la licencia de formatos de televisión para su posterior adaptación en el mercado global. En este contexto, la comercialización de formatos es vista como una respuesta de la industria para conciliar dos tendencias aparentemente contradictorias: la globalización, es decir, la necesidad de llegar a audiencias globales, y la preferencia de las audiencias por lo local, lo culturalmente próximo.

Pero el formato no es solo una moda o una oportunidad de la industria sino que es el síntoma de un cambio más profundo en la televisión. Hoy podemos referirnos a los programas de televisión —antes considerados contenidos con fuerte anclaje local o nacional — como productos estandarizados que pueden ser fácilmente trasladados de una cultura a otra o, incluso, ser pensados desde el inicio como productos globales (Bourdon, 2012, p. 11).

El análisis del mercado global de formatos no solo provee información acerca de las estructuras de producción y adquisición de contenidos televisivos, sino que también contribuye a responder preguntas acerca de la dinámica del negocio y el funcionamiento de la cadena de valor del productor de contenidos. Este es el propósito de esta investigación: estudiar los cambios generados en la dinámica del negocio provocados por la exportación e importación de formatos televisivos. Además, en el presente trabajo se propone como caso de estudio la estrategia de exportación de contenidos en el mercado argentino en los últimos veinte años. 
El enfoque asumido es interdisciplinar. Se busca integrar los estudios sobre estrategias internacionales de empresas de medios con las investigaciones sobre la circulación de formatos de televisión. La primera perspectiva - International Media Management - se detiene en lo relativo a la adopción de estrategias de exportación de contenidos por parte de las empresas de medios (Rohn, 2004). Por su parte, los TV Format Studies profundizan en el funcionamiento del mercado global de televisión, en particular en lo que se refiere a los mecanismos de adquisición y adaptación de los formatos en el mercado internacional (Moran, 2009a).

La investigación se centrará en el conocimiento pragmático de los actores específicos, es decir, los profesionales de la televisión involucrados en la producción y distribución de formatos de televisión en la Argentina. Las razones para poner el foco en la visión que tienen estos profesionales son dos: la primera se relaciona con la necesidad de reconocer la importancia de las prácticas industriales en la definición del medio televisivo (Oren y Shahaf, 2012, p. 6).

La segunda razón para centrar la investigación en el conocimiento de los profesionales es la inexistencia de trabajos previos sobre el mercado televisivo argentino que hayan adoptado una perspectiva de gestión o de negocio. Los únicos trabajos sobre la exportación de contenidos de televisión en este mercado son: el informe realizado por el Centro de Estudios para el Desarrollo Económico Metropolitano (Cedem) (2009) y el trabajo de Artopoulos, Friel y Hallak (2011) sobre la exportación de bienes diferenciados en países en desarrollo. Esta escasez de antecedentes conduce a que el presente trabajo tenga un carácter más bien exploratorio y descriptivo. No se pretende analizar todos los aspectos o factores que inciden en el desarrollo y la exportación de formatos de televisión en el país, sino solo aquellos que influyeron decisivamente en el posicionamiento de la Argentina como productor $y$ distribuidor internacional de contenidos televisivos en los últimos veinte años.

En el contexto latinoamericano, el mercado audiovisual argentino es un mercado desarrollado, no tanto por su tamaño, sino por el alto grado de acceso de la población a los productos de los medios, expresado, por ejem- 
plo, en índices de penetración en hogares del 98 \% para la televisión abierta y $87 \%$ para la televisión paga.

El sistema televisivo argentino cuenta con 49 canales, de los cuales cinco se ubican en Buenos Aires, la capital de país. Desde inicios de la década de los noventa hasta la actualidad, el canal 11 - Telefe - y Canal 13 se reparten el domino del mercado tanto en términos de audiencia como de inversión publicitaria. En el año 2013, Telefe, junto con Canal 13, recibían juntos el $60 \%$ de la inversión publicitaria destinada a televisión, seguidos del cable que recibe el $26 \%$, quedando el restante $14 \%$ repartido entre los otros tres canales de tv abierta: Canal 9, América TV y la TV Pública. Con respecto al volumen de inversión publicitaria la cifra estimada es de 600 millones de dólares anuales para la televisión abierta, y entre 80 y 100 millones de dólares para la televisión paga.

En los últimos quince años se evidenció una paulatina pero constante migración de contenidos y de la audiencia de la televisión abierta a otras plataformas como televisión por cable, satelital o Internet. Según IBOPE Media Argentina, en el periodo enero-junio de 2013 el cable concentraba el 43,5\% de la audiencia de televisión, seguido de Telefe con el 17,3\%, Canal 13 con el 15,7\%, mientras que Canal 9, América Tv y Tv Pública recibían el 10,2, 8,5 y $4,8 \%$ restante, respectivamente. Además, en la última década Argentina desarrolló la TDA, un modelo de distribución digital terrestre, que actualmente cuenta con 29 canales, seis de los cuales son televisión pública: Encuentro, Paka Paka, DeporTV, Tec TV, Incaa Tv y Canal 7-TV Pública.

Con respecto a la producción de contenidos audiovisuales, Argentina es un referente en la industria televisiva regional gracias a la reciente y exitosa exportación de productos y de servicios que posicionó al país entre los primeros exportadores de formatos de ficción. En el año 2013, la capacidad producción de Argentina fue aproximadamente de 15.000 horas anuales de programación, de las cuales alrededor de 2000 son exportadas al mercado internacional (E. Ferrara) ${ }^{3}$.

3 Se citarán de este modo las entrevistas en profundidad realizadas durante esta investigación por los autores. Un listado completo de las entrevistas indicando cargo, empresa y fecha de realización se encuentra después de las referencias. 


\section{Definición del formato de televisión y de su cadena de valor}

Un formato de televisión es una estructura explicativa original de cualquier tipo de programa, realizada con una articulación detallada y exhaustiva de sus fases secuenciales y temáticas, adecuada para la transposición en uno más productos destinados a la audiencia también a través de adaptación, elaboración, transformación o traducción (Fusco y Perrotta, 2008).

El desarrollo y la comercialización de formatos de televisión es un proceso complejo y abstracto en el que se distinguen al menos tres dimensiones: la instrumental, la económica y la narrativa o cultural. En la primera dimensión el formato se presenta como una tecnología de producción, un instrumento de trabajo para los productores de contenido.

Según Jensen (2008), el formato es un fenómeno relativamente abstracto compuesto por un número de entidades separadas y, al mismo tiempo, superpuestas:

- Idea o concepto: contexto, origen de la idea, precedentes, peculiaridades, claves de éxito.

- Paper format o descripción del formato: a la idea básica del programa se le suma la definición del género, de la audiencia a la que se dirige, duración, número de emisiones, etc.

- Tv format package: incluye, además del paper format, la denominada "biblia de producción" en la que se detalla la mecánica del programa, el perfil de los protagonistas, la escenografía, la iluminación, la música, los efectos sonoros, etc.

- Tv program format: cuando el programa ya ha sido producido en el mercado de origen se pueden compartir, además de los capítulos emitidos, el plan y el presupuesto de producción, los datos de rating o comportamiento de la audiencia en diferentes mercados, las acciones de marketing, etc. 
Desde esta primera perspectiva, el formato de televisión es definido como una fórmula o receta:

Un formato es como una receta. Para hacerlo bien, hay que medir todos los ingredientes cuidadosamente para luego seguir la receta paso a paso. Cuando se sigue cada fase de la receta según lo prescrito: ¡los resultados están garantizados! Un programa que ha sido un éxito de rating en un país tiene todas las posibilidades de tener el mismo éxito en otro país, sin errores costosos y sin incurrir en grandes costos de desarrollo (Saló, 2003, p. 33).

En la segunda dimensión, la económica, el formato es principalmente una mercancía, un objeto de protección, intercambio y acuerdos comerciales entre varios actores del proceso de producción: quiénes conciben el formato, quiénes lo venden, quiénes producen el programa y los responsables de la emisora o plataforma donde se emite.

Desde esta segunda perspectiva, el formato es definido como un intento de la industria por materializar y comercializar una idea que es por naturaleza inmaterial (Jensen, 2007, p. 22). La idea o el concepto son punto de partida del formato pero desde el punto de vista legal la idea no es suficiente para proteger el proyecto. Para que una idea sea protegible, además de ser original, debe estar lo suficientemente desarrollada y expresada como formato (Guerrero, 2010).

Por último, en su tercera dimensión, la narrativa, el formato es una forma de diseñar experiencias narrativas, un modo particular de generar representaciones sociales de la realidad. En este caso, el formato es visto como una matriz que organiza contenidos, articula géneros y arquetipos narrativos vinculados a una cultura determinada.

Desde esta tercera perspectiva, la creación y circulación de formatos está estrechamente vinculada a los procesos de globalización de la cultura y su contrapunto: la necesidad de adaptar contenidos globales a factores locales (Bielby y Harrington, 2008). Los formatos permiten minimizar el "descuento cultural" ya que son, al mismo tiempo, textos transparentes — tienen elementos fácilmente reconocibles y asimilables por las audien- 
cias-y opacos - contienen elementos locales que los hacen especialmente atractivos-(Jensen, 2007, p. 26).

La mayoría de los trabajos sobre formatos de televisión se detienen solo en una o dos de las tres dimensiones, a pesar de que en la realidad estos aspectos están fuertemente integrados. Un primer grupo de trabajos lo constituyen aquellos cuyo objetivo es analizar los procesos de desarrollo y de adaptación de los formatos. El énfasis está en la dimensión cultural e instrumental del formato ya que se trata de detectar las diferencias culturales entre los países de origen y de destino del formato, y de explicar el modo en que estas diferencias fueron - con mayor o menor éxito- minimizadas en la versión local. Una excelente compilación de trabajos que adoptan esta mirada se encuentra en Tv Formats Worldwide. Localizing Global Programs (Moran, 2009b) y Global Television Formats: understanding television across borders (Oren y Shahaf, 2012).

Un segundo grupo de trabajos es el que se detiene a analizar las estructuras del comercio internacional: qué organizaciones participan y de qué modo se exportan o importan los contenidos. En estos estudios el foco está en la dimensión económica de los formatos de televisión y su propósito es explicar las estrategias desarrolladas por las empresas para entrar en otros mercados a través del contenido desde una perspectiva de gestión o de negocios (Bielby y Harrington, 2008; Moran, 2009a; Rohn, 2010).

En la presente investigación se busca considerar a los formatos de televisión en sus tres dimensiones, con un énfasis claro en las dimensiones instrumental y económica. Un trabajo que puede ser considerado como antecedente es el análisis de la cadena de valor del formato de televisión propuesto por Lantzsch, Altmeppen y Will (2009), y el mencionado informe de Artopoulos et al. (2011).

Para Lantzsch et al. (2009), el desarrollo y la comercialización internacional de formatos es más que una transacción o licencia de producto, es un proceso complejo y dinámico en el que se distinguen claramente tres etapas: la etapa de desarrollo y producción en el país de origen, la etapa de distribu- 
ción o adquisición del formato, y la etapa de adaptación o reproducción en la que el formato es producido y emitido en el país de destino.

En cada una de estas etapas intervienen muchos actores, cada uno de los cuales es capaz de desempeñar más de una función. Por ejemplo, la fase de desarrollo del formato puede depender del autor de la idea original, pero también del productor $\mathrm{o}$, incluso, del distribuidor que contrata a un autor para desarrollar un formato. Algo similar ocurre con la distribución del formato: el licenciatario puede ser un autor, una productora, un canal de televisión o una empresa comercializadora de derechos.

Esta multiplicidad e intercambiabilidad de los actores da lugar a una serie de complejas redes interorganizacionales que demandan en las empresas y en los profesionales una capacidad de gestión y de negocios centrada más en la generación de relaciones personales y en la capacidad de comunicarse a nivel global que en contratos formales (Lantzsch et al., 2009, p. 93).

Desde una perspectiva que intenta combinar la mirada del management con la de los estudios de televisión, puede decirse que cuando una empresa adopta una estrategia de entrada en mercados extranjeros enfrenta como uno de sus principales desafíos la adaptación de sus productos y sus procesos de producción de contenidos a los estándares y la dinámica del mercado internacional (Artopoulos et al., 2011, p. 51). Si esta entrada es además a través de los contenidos - productos consumidos principalmente por su carácter simbólico-, el desafío es aún mayor ya que junto a las barreras económicas, políticas o legales es muy posible que se encuentre con barreras y diferencias culturales que pueden dificultar la aceptación del producto en el mercado de destino. Estas barreras se refieren no solo al contenido en sí sino también al capital o bagaje cultural de las audiencias y a los estilos de producción de los contenidos (Rohn, 2010).

En este contexto, el desarrollo y la comercialización de formatos de televisión pueden ser considerados como una estrategia de la industria para minimizar las barreras culturales de entrada de sus productos en mercados extranjeros. Esta estrategia es particularmente interesante en el caso de los países que históricamente no se han caracterizado por un amplio desarrollo de 
la exportación de contenidos pero que en los últimos años y gracias a la aparición de los formatos de televisión han ganado protagonismo. Como señala Torres (2012), en las últimas dos décadas una serie de cambios en la tecnología y en los sistemas de distribución de contenidos, sumados también a algunos cambios en la regulación que favorecieron la producción de contenidos locales, facilitaron el crecimiento de nuevos jugadores en el mercado de formatos como es el caso de Israel, China, Brasil y, más recientemente, Argentina y Colombia. Como indicador de este mayor desarrollo pueden tomarse algunas cifras publicadas en informes de la Format Recognition and Protection Association (Frapa) y de la consultora The Wit.

De acuerdo con el informe Tv Formats to the World (Frapa, 2009), en el mundo el volumen generado por la comercialización internacional de formatos de televisión fue de aproximadamente 9,3 mil millones de euros. Esta cifra se estima que ha sido duplicada en el periodo 2009-2013, alcanzando los 19 mil millones de euros en el año 2014 (E. Geneste). Los tres países líderes en el mercado en función del número de formatos importados y exportados en el periodo 2006-2008 son: Reino Unido, con un total de 66 formatos importados y 275 exportados; EE.UU., con 116 importados y 159 exportados, y Holanda, con 103 importados y 63 exportados.

La mayoría de los formatos distribuidos internacionalmente son nonscripted o formatos basados en procesos (Oren, 2012, p. 368). Los géneros más frecuentes en este segmento del mercado son: game shows, quiz shows, variety shows, talentor casting shows, talk shows, reality shows y factual shows. Un segundo segmento es el formado por los scripted formats o formatos basados en narrativas, en el que se inscriben los géneros: telenovela, dailysoap, drama, sitcom, sketch shows y scripted reality shows. Este tipo de formatos son especialmente relevantes para esta investigación ya que, como se verá luego, estos son los géneros en los que los países latinoamericanos se han destacado y a través de los cuales han logrado exportar al mundo mayor número de formatos.

Como indicador de esta mayor relevancia de los formatos guionados originados en América Latina, en el mercado internacional pueden considerarse algunos números publicados en el estudio de mercado Success Stories 
Arround the World. The WIT Guide to Scripted Formats 2014. En el informe la consultora señala que en los últimos diez años, y a nivel internacional, el número de series locales basadas en formatos guionados extranjeros aumentó significativamente de 21 series en 2003 a 67 series en 2010 y 96 en el año 2013. En este segmento, los países con mayor número de formatos vendidos históricamente son Inglaterra, con 79 formatos guionados, seguida de Estados Unidos con 72, Argentina con 52, España con 36 y Colombia con 31. En los últimos años crecieron también los formatos comercializados en mercados extranjeros por Israel, Australia y Alemania. Los países que compran mayor cantidad de formatos guionados — según el mismo informeson Estados Unidos y Rusia, seguidos, en especial en los últimos años, por Colombia, México y Francia (TheWit, 2014).

\section{Metodología y preguntas de investigación}

La metodología utilizada es cualitativa. En el estudio de la información disponible sobre el desarrollo y la exportación de formatos en Argentina, se consideran las descripciones y las explicaciones obtenidas en las entrevistas en profundidad realizadas a tres grupos de profesionales. El primer grupo es el integrado por los responsables de distribución internacional de contenidos que trabajan en productoras, emisoras o en empresas dedicadas exclusivamente a la comercialización de derechos de distribución. El segundo grupo está integrado por productores independientes y por programadores de canales de televisión, y el tercer grupo por abogados especializados y consultores de mercado.

Se realizaron un total de 30 entrevistas, en un periodo de dos años —abril de 2012 a julio de 2014-, y las empresas y los profesionales fueron seleccionados con un doble criterio: la experiencia de la empresa y la trayectoria del profesional en la producción o distribución de formatos de televisión. Entre las productoras y distribuidoras de contenidos audiovisuales seleccionadas para las entrevistas se encuentran, por orden alfabético: DoriMediaGroup, Endemol Argentina, Ideas del Sur, Mediabiz, Polka, RGB Entertainment, Resonant TV, Telefe S.A., Televisa Internacional, Walt Disney Latinoamérica y Wintv. Entre las consultoras y los estudios de abogados: Alesina y Asociados, Biset\& Chamorro, HD International Consul- 
ting, Noujau International Consulting, Prensario Internacional. Además, se entrevistaron algunos productores independientes y representantes de la Fundación ExportAr y de Frapa.

Las entrevistas se realizaron en el lugar de trabajo habitual del entrevistado y tuvieron una duración de entre 60 y 90 minutos. El objetivo de estas entrevistas fue obtener una descripción de los procesos de desarrollo, producción y distribución internacional de los contenidos televisivos para detectar cuáles fueron los cambios introducidos en estos procesos a partir de la exportación e importación de contenidos, ya sean latas, formatos o servicios de producción.

El análisis de la información recogida en las entrevistas se organiza en torno a tres preguntas directamente relacionadas con el objetivo de esta investigación. La primera, es acerca de los factores que motivan a las productoras o emisoras de televisión a desarrollar estrategias de entrada en mercados extranjeros a través del contenido, ya sea por medio de la venta de latas, la licencia de formatos o la prestación de servicios de producción. La segunda, se dirige a las demandas o exigencias que genera la adopción de una estrategia de internacionalización sobre los productos y sobre los procesos de desarrollo, producción y comercialización. La tercera pregunta se refiere a la función que cumplieron los formatos de televisión en este proceso de internacionalización y apunta a detectar qué desafíos y qué oportunidades generó el desarrollo y la comercialización de formatos de televisión en la cadena de valor y en la dinámica del negocio de la industria.

A continuación, y organizadas en torno a estas tres preguntas, se presentan las respuestas recogidas en las entrevistas y se citan a modo de ejemplo e ilustración algunos casos o algunas afirmaciones de los entrevistados.

\section{Argentina: contenidos para exportar}

\section{La apertura del sector: razones para exportar}

Según los productores y distribuidores entrevistados, los principales factores que motivaron a las productoras o emisoras de televisión a desarro- 
llar estrategias de entrada en mercados extranjeros a través del contenido fueron: a) la necesidad de encontrar formas alternativas de cubrir los costos de producción locales, b) la reconocida creatividad y habilidad de los productores argentinos para trabajar en equipo con presupuestos y tiempos muy acotados, y c) la experiencia de los productores en la adaptación de contenidos extranjeros.

Desde comienzos de los años cincuenta la estrategia habitual en el sector televisivo era la venta de latas — programas terminados-, y de los libros de las novelas, para su posterior adaptación a otros mercados. Esta experiencia de venta de guiones de novelas es común a la desarrollada en otros países de la región. Como señala Straubhaar (2012), el flujo de guiones de telenovelas en Latinoamérica precedió la explosión de la venta de latas de Estados Unidos en los años sesenta (Straubhaar, 2012, p. 173).

Como ejemplo claro de esta estrategia puede citarse el caso de Muñeca brava, telenovela producida por Raúl Lecouna y Telefe, transmitida por el canal durante 1998 y 1999, con un enorme éxito en la pantalla local. En los años siguientes la novela fue vendida como lata a más de sesenta países y se hicieron además las siguientes adaptaciones: Anjo Salvagem, producida por TVI en Portugal; Al diablo con los guapos, producida por Televisa en México; La Tayson corazón rebelde, producida por Frecuencia Latina en Perú; Millie, producida por Star en la India, y Hiafizaa, producida por MD Entertainment en Indonesia (E. Borensztein).

A comienzos de los años noventa, la necesidad de cubrir los costos de producción local llevó a los productores a buscar otras fuentes de financiación que no fueran solo las de la venta de publicidad y la venta de latas o de libros. Esta búsqueda de alternativas estuvo vinculada tanto al tamaño relativamente pequeño del mercado argentino como a la alta sensibilidad de la inversión publicitaria hacia los cambios en el contexto económico general.

$\mathrm{Al}$ respecto, señala uno de los empresarios entrevistados:

A pesar de que la Argentina tiene gran talento, la producción no puede ser cubierta con el mercado local. La torta publicitaria no alcanza 
sino que hay que ir a otros negocios, hay que salir afuera. La causa de la exportación es que tenemos tan buen talento que podemos salir a exportarlo. La paradoja es que aquí ese talento solo nos sirve para cerrar las cuentas. Es un negocio con mucho riesgo, con baja masa crítica. Entonces, se debe ampliar la masa distribuyendo el riesgo. Hoy, cuando exportamos un formato, le estamos sacando un porcentaje a la venta para cerrar la cuenta local. A veces hay un gran hit, como fue el caso de Patito feo, y eso permite tener un extra (E. Lagomarsino).

Una primera alternativa fue la desarrollada — por tomar solo un ejemplo- por el programa Chiquititas, una serie infanto-juvenil producida por Cris Morena Group y emitida por Telefe entre 1995 y 2001. En el año 2006 se estrenó una nueva versión de la serie. En total se filmaron 1112 episodios. En 1998 la serie ganó el Martín Fierro al mejor programa infanto-juvenil, y en 2001 se estrenó en cine la película Chiquititas: rincón de luz. La lata de la serie fue vendida a 32 países y en el periodo 2007-2008 se hicieron versiones de la novela en Portugal, Rumania, México y Brasil. En el año 2013 Brasil hizo una nueva versión.

Lo novedoso de este proyecto desde el punto de vista del negocio fue la búsqueda de ingresos alternativos y complementarios a los de la pauta publicitaria. A la venta de licencias se le sumó la venta de música, revistas, teatro y diferente tipo de merchandising - desde ropa hasta cepillos de dientes - asociados al contenido del programa. Estos ingresos alternativos llegaron a representar entre el 25 y el $30 \%$ del costo de producción del programa y les dieron a los productores un expertise en la gestión de licencias de producto (E. González).

Una segunda alternativa desarrollada por los productores fue la venta de servicios de producción para formatos extranjeros. El factor que posibilitó el desarrollo de este negocio fue la experiencia en producción diaria, el prestigio y la habilidad de los productores argentinos para gestionar el proceso y los equipos humanos de producción. Un ejemplo de esta capacidad fue la producción para diferentes mercados del formato Expedición Robinson, conocido también como Survivor, un formato de entretenimien- 
to creado por Charlie Parsons, desarrollado por Mark Burnett. La empresa argentina Promofilm, después de licenciar y producir la versión argentina en los años 2000 y 2001, decidió aprovechar la experiencia y ofrecer a los otros países - Sudáfrica, Dinamarca, Finlandia y muchos otros- el servicio de producción del formato.

Así lo explica uno de los protagonistas del proyecto:

Cuando licenciamos el programa había salido en muy pocos países y se logró armar un grupo de gente con buena experiencia. Conseguimos la locación —Boca del Toro, en Panamá-y tener todo listo para hacer el programa en poco tiempo: las mismas instalaciones, los mismos juegos. Así fue como — por ejemplo- los turcos, los vascos, los rumanos, licenciaban el formato original y luego lo producíamos nosotros. Podemos decir que lo que ofrecíamos era un formato de producción (E. Muzslaski).

En otros casos, las producciones se hacían en Argentina para ser vistas en el extranjero, como fue por ejemplo el caso del formato WipeOut, de origen japonés. La versión estadounidense fue producida en Argentina por Endemol. Una vez construido el parque acuático para realizar la versión argentina del programa - Hombre al agua-, se hicieron allí diferentes versiones para países tan distantes como Rusia o Rumania.

Una tercera alternativa explorada fue la licencia de formatos de televisión extranjeros para la pantalla local, como una manera de disminuir el riesgo en la inversión - apostando por productos ya probados en otros mercados - y, al mismo tiempo, de ganar experiencia en los procesos de adaptación de formatos a las necesidades de otra audiencia. Como señalan Waisbord y Jalfin (2009), desarrollar y producir contenidos en y para otros mercados geográficos exige aprender a detectar y extraer del contenido aquellas barreras culturales que dificultan la entrada y la aceptación por parte del público de un producto de origen extranjero. Esta habilidad es necesaria tanto para "deslocalizar" los formatos argentinos para que puedan ser ofrecidos en el mercado internacional como para adaptar formatos extranjeros a las demandas de la audiencia argentina. En este sentido, la producción local a comienzos de los años noventa, de las sitcoms Married with Children, 
Who is the Boss, The Nanny o Desperate Housewives le dio a los productores argentinos un conocimiento mayor de las diferencias culturales, tanto las relativas al contenido como al estilo de producción (E. Di Guglielmo).

Un ejemplo de este tipo de aprendizaje se encuentra en el caso de $\mathrm{La}$ Niñera, una coproducción de Telefe Contenidos y Sony Television de la serie de 1993 de Fran Drescher, que si bien ya había sido realizada fuera de Estados Unidos —en Turquía, Rusia y Grecia—, se realizó por primera vez en un país hispanohablante. Además de la necesidad de adaptar los guiones y los contenidos a la sensibilidad y el sentido del humor de la audiencia argentina, los productores requirieron un entrenamiento especial de los actores que no estaban acostumbrados a las exigencias del sitcom, un género que no permite la improvisación de los diálogos sino que requiere el ensayo y la fidelidad al guión (Cecinini, 2005). Además, mientras que en la sitcom los personajes suelen tener más peso que la historia misma — su estructura es episódica-, en la comedia o serie argentina el personaje crece o disminuye a medida que avanza el relato, se cuenta con el largo plazo (Basani, 2005).

Las tres estrategias mencionadas: venta de libros, venta de servicios de producción y adaptación de formatos extranjeros fueron los primeros pasos en el desarrollo de una estrategia internacional por parte de los productores y distribuidores de contenidos de Argentina. Esta adopción de lo que Artopoulos et al. (2010) denominan un "modelo exportador de contenidos”, tendrá como siguiente etapa el desarrollo y la licencia de las novelas no como libros o latas sino como formatos guionados.

Uno de los primeros formatos guionados exitosos fue la miniserie de 24 capítulos Los Simuladores (2002) que abrió para Telefe el mercado de México, además de venderse como formato a Chile, España y Rusia, con una activa participación de Telefe en las versiones locales. La siguiente apuesta fue Resistiré (2003), la primera novela de Telefe pensada para el prime time, que incluía a la audiencia masculina a través del suspenso y la acción. Al año siguiente, la novela Los Roldán — producida por la empresa Ideas del Sur — buscaría también al mismo público pero a través del humor. 
En este caso, el producto no podía exportarse como lata ya que el contenido era muy localista, pero como formato fue vendido a Grecia, Colombia, Chile, México, El Salvador, entre otros. En los años 2005 y 2006, otras novelas con gran aceptación como formatos en el mercado internacional fueron Montecristo y Amor en Custodia. En ambos casos la asesoría autoral y de producción fue clave para concretar la venta del formato y adaptar exitosamente la historia (E. Wasserman).

\section{Cambios en los procesos de desarrollo, producción y comercialización}

Según se desprende de las entrevistas realizadas, la licencia internacional de latas, formatos y servicios de producción generó la aparición de una serie de desafíos para los responsables del desarrollo, la producción y la comercialización de contenidos, entre ellos los más importantes son: a) la adopción de estándares de calidad internacionales, b) el aprendizaje sobre la negociación de derechos de distribución, y c) el desarrollo de nuevos mercados.

En primer lugar, la apertura del mercado obligó a los productores a revisar y adaptar la calidad técnica y los procesos de producción a los estándares internacionales. Esta necesidad de ajuste abarcó desde la duración de los capítulos de las series — necesidad de que tengan una duración uniforme-, hasta la planificación más detallada de los contenidos de los programas y un control presupuestario más estricto. En palabras de un productor, y por citar solo un ejemplo:

Cuando empezamos a trabajar para afuera fue necesaria una reconversión cultural. Por ejemplo, el canal extranjero nos pedía de antemano un resumen de cada uno de los programas de viajes que haríamos para ellos. Querían saber qué verían programa por programa. Eso nosotros no lo hacíamos así, se pensaba en los primeros capítulos y después se iba improvisando o viendo sobre la marcha, según fuera la respuesta del público. Además, en el ámbito internacional los controles presupuestarios son muy precisos. En ese momento - los noventa - en la Argentina se trabajaba sin presupuesto, era otro mundo. Hoy, sin un control minucioso no funciona. Podemos decir que hubo una reconversión del productor en gestor, en administrador (E. Kon). 
En segundo lugar, la salida al mercado internacional reclamó un desarrollo de las capacidades de negociación de los derechos de distribución y una supervisión constante de los cambios tanto de la oferta como de la demanda de productos y servicios en el mercado internacional. Esta habilidad es especialmente necesaria en un contexto en el que los compradores tienen con frecuencia mayor poder de negociación que los vendedores argentinos ya sea por su trayectoria o por contar con una cadena de valor integrada, con productoras y canales de televisión propios. Como ejemplo, un distribuidor explicaba cómo había sido la venta de los primeros formatos argentinos a México:

\begin{abstract}
Al comienzo nos pasó que vendimos el formato Rebelde Way a México y lo vendimos con todos los derechos. Cuando íbamos a las ferias a vender nuestra lata nos decían que ya tenían la versión mexicana y no les interesaba la nuestra. Incluso en Venezuela sucedió que tenían la lata argentina en un canal y la mexicana en el otro. Nos sirvió de aprendizaje: ahora hacemos todo lo posible por retener los derechos de la nueva versión, aunque no siempre lo logramos, ya que eso depende del tamaño del distribuidor o la productora que licencia el formato (E. Wasserman).
\end{abstract}

Esta negociación de los derechos se hizo más compleja en los últimos años con la aparición de nuevas plataformas de distribución. Además de contenidos para el cable y el video on demand, los productores demandan contenidos adaptados a la Internet y a plataformas móviles, con aplicaciones — por ejemplo juegos_ que permitan a la audiencia interactuar con los contenidos (E. Antonini).

En tercer lugar, la decisión de desarrollar y exportar formatos guionados permitió la llegada de los contenidos argentinos a nuevos mercados como por ejemplo, el latinoamericano, que hasta entonces no aceptaba contenidos con acento argentino (E. Di Guglielmo). Una de las claves del éxito de los productores y distribuidores argentinos señalada en las entrevistas es la capacidad para construir relaciones de confianza con los compradores, ofreciéndoles un tratamiento personalizado y servicios adicionales como la asesoría autoral o el envío de productores para colaborar en la versión local. Tal fue la experiencia de Telefe cuando comenzó a vender sus contenidos en el mercado mexicano: 
En Telefe no teníamos ningún negocio con México y hoy es para nosotros uno de los territorios más importantes. Empezamos con Azteca y como con los formatos tenían dudas les ofrecimos llevar el productor, el director, un equipo nuestro, que iba a estar un tiempo para ver ciertas cosas que importan y fue un éxito. Fue un prime time en el que como muy pocas veces sucede Azteca le ganó a Televisa. Eso nos abrió las puertas y nos vio Televisa, que no sabía quiénes éramos. Una vez que generaste la confianza, todo se facilita. Todo se sabe, esta es una industria de mucho contacto personal (E. Parra).

Esta habilidad de los actores para explorar nuevos mercados es definida por productores y distribuidores como una necesidad, el único modo de sobrevivir en un entorno muy cambiante donde los países que hoy son compradores de latas pueden empezar a comprar formatos o convertirse incluso en productores y vendedores. El ejemplo de esta posibilidad de cambio de roles más citado en las entrevistas es el de Turquía. En palabras de un distribuidor:

Turquía empezó comprando "latas latinas"; después, formatos; después contrató guionistas latinos y ahora produce por encima de la calidad de la Argentina, a pesar de que tienen un costo de producción de telenovela mucho más alto. Alguien que era un comprador hoy es un competidor. A Turquía lo tenemos como fuerte competidor en Europa del este. La torta publicitaria de Turquía es más grande que la de Argentina. Por eso pueden producir con costos de producción tan altos (E. Lagomarsino).

\section{Desafíos y oportunidades generados por los formatos de televisión}

En el proceso de internacionalización, y según los productores y distribuidores entrevistados, el desarrollo y la comercialización de los formatos de televisión tuvo la función de dinamizar la industria y generar una mayor conexión entre los distintos actores de la cadena de valor. En este contexto, los principales desafíos y las oportunidades planteadas por los formatos fueron: a) la construcción del formatpackage; b) el desarrollo de nuevos tipos de alianzas colaborativas entre empresas nacionales y extranjeras, y c) la necesidad de profundizar el diálogo y la integración entre los diferentes actores. 
El desarrollo y la licencia del formato exigió a los autores y productores crear contenidos con una trama firme, bien estructurada y que - al mismo tiempo- admita variaciones, que pueda adaptarse a otros contextos. Además, en muchas ocasiones la licencia de un formato demanda a autores y productores extraer "la fórmula" de series, novelas o programas de entretenimiento que llevaban años en el mercado o que habían sido pensados solo para la pantalla local. Por ejemplo, en el caso del formato $C Q C$, creado en 1995 por la productora Cuatro Cabezas y exportado ya a más de diez países, la construcción del formato es narrada por uno de sus protagonistas del siguiente modo:

\begin{abstract}
Cuando nos llamaron para comprarnos el formato no teníamos ni un papel. Empezamos por hacer una descripción de los personajes de la mesa y de los periodistas que salían a hacer exteriores. Al darle eso al productor, le permitíamos preparar a su gente. Después, le dábamos un menú de todas las notas que debían hacerse en las primeras semanas, por ejemplo: lograr que, cuanto antes, te echen del Parlamento. Además, compilamos todas las excusas de por qué CQC no podía hacerse en un determinado país, por ejemplo "porque no tenemos conductor", y dábamos respuestas sobre cómo solucionarlas. Así fue como se fue construyendo el formato y la biblia de producción (E. Kon).
\end{abstract}

Una oportunidad abierta en los últimos veinte años tras la aparición de los formatos fue el desarrollo de diferentes tipos de alianzas colaborativas entre las empresas productoras y distribuidoras nacionales y extranjeras: desde la coproducción o cofinanciación de un proyecto hasta el acuerdo de distribución del formato o la oferta del servicio de producción, ya sea en Argentina o en otro país. Como ejemplo de la diversidad creciente de estos acuerdos puede citarse el caso de Los exitosos Pells, proyecto coproducido por Underground y Endemol Argentina, emitido y distribuido por Telefe, o también el caso de En Terapia, formato de ficción israelí cuya versión argentina fue coproducida en Argentina por Dori Media y la TV Pública.

Este proceso de apertura y flexibilización es resumido por uno de los directivos entrevistados del siguiente modo:

Nosotros éramos muy de mirarnos el ombligo. La creatividad tenía que surgir de dos o tres personas, en donde compartir o buscar la 
creatividad ajena era mala palabra. Hay que romper estas barreras de egocentrismo cultural, lo que es muy difícil. Sin matar la gallina de los huevos de oro que es nuestra pantalla local, tenemos que tratar de encontrar propuestas de valor para los anunciantes, establecer conversaciones con los clientes, y asumir el enorme desafío de instalarnos en el mundo de habla hispana. Si queremos ser una de las compañías más admiradas de producción de contenidos multiplataforma, a la venta de la lata y el formato - que seguiremos explorando- debemos sumar el concepto de las alianzas, de las coproducciones y de distintas formas de revenue sharing, en donde todos ganemos un poquito (E. Wahener).

Para poder desarrollar exitosamente este tipo de alianzas las empresas productoras de contenidos enfrentan algunos desafíos. El primero y tal vez el más importante es la dificultad de los creativos, programadores y productores ejecutivos para percibir que su cadena de valor incluye a los distribuidores y a los responsables de venta internacional. Aun cuando la mayoría de los productores entrevistados son conscientes de una necesidad de diálogo y colaboración entre las distintas fases del proceso de creación, producción y comercialización de un contenido en otros mercados, en la práctica los creativos y programadores tienden a pensar solo y principalmente en la pantalla local, y a considerar a la exportación de contenidos como un paso posterior e independiente, que en nada debería interferir en su trabajo creativo.

Por su parte, para los distribuidores, el desarrollo y la exportación de latas, formatos y de servicios de producción exige a los responsables de vender los contenidos en el mercado internacional una participación activa en los procesos de creación y de producción de los mismos, con el objetivo de ayudar a minimizar o, al menos, anticipar las barreras culturales que puedan encontrar los productos al llegar a otras audiencias. Esta necesidad de diálogo y colaboración aumenta cuando el contenido se piensa desde el primer momento para el mercado internacional, como ocurrió con el caso de Lalola, producida por Underground y Dori Media, y vendida como lata y como formato un total de 135 veces en 110 territorios.

Lo que nos jugó a favor en Lalola es que se tuvo mucho cuidado a nivel de la producción. Empezando por el léxico: le quitamos todos los 
modismos argentinos. Además, se apuntaba a un producto familiar: no había malas palabras, escenas de sexo, etc. También había que trabajar mucho con los actores para que entendieran el tipo de diálogos que necesitábamos. El idioma era importante, especialmente para vender la lata en Latinoamérica (E. Antonini).

Este diálogo entre creadores y productores es además clave cuando se trata de adaptar la extensión de la novela a las necesidades del prime time en otros mercados geográficos. Siguiendo con el caso de Lalola, sucedió que países de Europa occidental como Portugal o Italia querían una novela de frecuencia semanal de 52 capítulos. La adaptación de la original - que tenía 150 capítulos de emisión diaria - la hizo la productora local pero con la asesoría del autor y productor argentino, ya que había que decidir qué líneas narrativas incidían en la historia principal, cómo podía hacerse el recorte. Una situación inversa se dio con la venta de la novela en Rusia, donde debido al éxito llegaron a hacerse 360 capítulos, más del doble de la versión original.

\section{Consideraciones finales}

Los formatos son una gran ayuda para comprender cómo la televisión oscila constantemente entre lo doméstico, lo comunitario, lo nacional, lo transacional y lo global. En términos de su funcionalidad y de la retroalimentación que generan entre lo convencional y lo innovador, lo local y el mundo mediatizado, el formato de televisión global es hoy la televisión en estado puro (Oren, 2012, p. 379).

La circulación de formatos de televisión se presenta como un campo de investigación apropiado para comprender el funcionamiento del mercado audiovisual, marcado en las últimas décadas por la creciente tensión entre dos fuerzas aparentemente contrarias: la globalización y la localización o preferencia de las audiencias por lo local.

El estudio de las estrategias de entrada en el mercado internacional, desarrolladas en los últimos veinte años por las empresas audiovisuales en Argentina, permite señalar algunos aprendizajes vinculados a una nueva dinámica del negocio televisivo, definida no solo por la creciente interac- 
ción e interdependencia entre empresas productoras, canales de televisión y distribuidoras o comercializadoras de derechos, sino también por la oferta de nuevos tipos de productos y de servicios.

A continuación, a modo de síntesis, se recogen algunos aciertos o claves que explican el éxito que encontraron las empresas argentinas para entrar y posicionarse con éxito en el mercado internacional de contenidos televisivos.

En primer lugar, y en relación con lo que al comienzo del artículo definimos como dimensión económica del formato, las empresas argentinas adoptaron una estrategia que podría denominarse de "productor boutique", ya que apostaron por la oferta de pocos títulos — condicionados por el tamaño relativamente pequeño del mercado - pero con una buena calidad de realización y un servicio muy personalizado, adaptado a las necesidades de las empresas productoras o distribuidoras extranjeras. Puede decirse que el énfasis no estaba en la originalidad o creatividad de la historia — difícil de predecir y de evaluar-, sino en el servicio que ofrecían a la empresa que incluía tanto la asesoría autoral como la transferencia del know how de producción de la serie o la novela. En esta línea, como pequeños productores buscaron un nicho en el cual poder diferenciarse: la oferta de la novela como formato de televisión. En esto se adelantaron a quienes eran hasta el momento los grandes productores de telenovelas en la región, acostumbrados a vender sus productos como libros o programas terminados. Además, en lo que se refiere a la dimensión narrativa del formato, puede señalarse que al ofrecer la novela como formato los productores argentinos pudieron superar también las barreras culturales que tenían los contenidos nacionales cuando intentaban viajar dentro de América Latina, no solo por las diferencias en el uso del español, sino también por las diferencias en el capital cultural de las audiencias.

En segundo lugar, y desde una perspectiva instrumental del formato, las empresas argentinas mostraron una gran flexibilidad $y$ adaptabilidad en los procesos de desarrollo, producción y comercialización de contenidos. A partir de la experiencia en la adaptación de formatos extranjeros, los productores argentinos se amoldaron a los estándares internacionales, tan- 
to en lo que se refiere a la calidad técnica como a los procesos de producción, facilitando así la creación de una marca y de un prestigio que facilitó la entrada de los contenidos argentinos en nuevos mercados, en especial en aquellos cultural y geográficamente más distantes. Esta flexibilidad en los procesos se notó también en el modo de comercializar los contenidos, traduciéndose en una participación creciente y activa en las ferias internacionales, con una apuesta fuerte por la construcción de relaciones de confianza con los distribuidores o productores internacionales.

En tercer lugar, y como corolario de la apuesta por el servicio y de la flexibilidad en los procesos antes señalados, las empresas argentinas supieron adoptar una visión global del negocio y pasaron, en relativamente poco tiempo, de considerar a la exportación de contenidos como una fuente de ingresos adicional, a verla como una oportunidad u ocasión para movilizar y dinamizar a toda la industria abriendo el juego a diferentes tipos de alianzas estratégicas entre productores nacionales y extranjeros. Esto es especialmente interesante si se considera que la primera salida de los contenidos argentinos al mercado internacional tuvo como motivación principal la necesidad de las empresas de cubrir los altos costos de producción locales. Es posible pensar que fue justamente esta urgencia por buscar fuentes de financiación adicionales a la publicidad local ofrecida la que movilizó a los productores a adaptar sus productos y procesos a los cambios en la oferta y la demanda del mercado internacional: de Europa a Estados Unidos y América Latina, de venta de latas a licencia de formatos y servicios de producción.

Por último, y a modo de cierre, parece oportuno señalar que el desarrollo y la comercialización de formatos de televisión habilitó una mayor articulación y un diálogo más fluido entre los diferentes actores de la cadena de valor, en particular entre productores y distribuidores que se encontraron con el desafío de pensar y desarrollar juntos una serie de nuevos productos y servicios. Esta oportunidad de crecer en integración y coordinación dentro de la cadena de valor del productor de contenidos, junto a la mencionada necesidad de pensar en nuevos tipos de alianzas estratégicas entre empresas productoras y distribuidoras nacionales y locales, sobrepasa los límites de la industria de la televisión, se extiende a toda la industria 
de los medios y el entretenimiento, y desafía tanto a los profesionales que trabajan en la industria como a los académicos interesados en comprender el funcionamiento y la dinámica del negocio en este sector.

\section{Referencias}

Altmeppen, K., Lantzch, K. y Will, A. (2005). Flowing Networks in the Entertainment Business Organizing International TV format Trade. The International Journal on Media Management, 9 (3), 94-104.

Artopoulos, A., Friel, D. y Hallak, J. (2011). Export Emergence of Differentiated Goods from Developing Countries: four Argentine Cases. Buenos Aires: Universidad de San Andrés.

Basani, Lorena (10 de febrero de 2005). Telefé adapta comedias de la tv norteamericana para enfrentar a Tinelli. Clarin. Recuperado de: http:// edant.clarin.com/diario/2005/02/10/conexiones/t-919189.htm.

Bielby, D. y Harrington, C. L. (2008). Global TV. Exporting Television and Culture in the World Market. New York: New York University Press.

Bourdon, J. (2012). Fromdiscret adaptations to hard copies. The rise of Formats in European Television. En Oren, T. y Shahaf, S. (eds.) (2012). Global Television Formats: Understanding Television Across Borders (pp. 112-127). New York and London: Routledge.

Cecinini, A. (20 de abril de 2005). El humor en tiempos de sitcom. La Nación. Recuperado de: http://www.lanacion.com.ar/697618-el-humor-en-tiempos-de-sitcom.

Cedem (2009). La exportación de contenidos y servicios de producción televisiva en la Ciudad de Buenos Aires. Buenos Aires: Observatorio de Comercio Internacional de la Ciudad de Buenos Aires.

Frapa (2009). The Frapa Report 2009. TV Formats to the World. Huerth: Frapa. 
Fusco, S. y Perrota, M. (2008). Rethinking the Format as a Theoretical Object in the Age of Media Convergence. Observatorio Journal, 7, 89-102.

Guerrero, E. (2010). El desarrollo de proyectos audiovisuales: adquisición y creación de formatos de entretenimiento. Comunicación y Sociedad, 23 (1), 237-273.

Jensen, P. (2007). Television Format Adaptation in a Trans-national Perspective. An Australian and Danish Case Study. Aarhus University. Recuperado de: http://nordicom.statsbiblioteket.dk/ncom/ files/16573/pm_jensen--tv-format_adaptation.pdf

Lantzsch, K., Altmeppen, K. y Will, A. (2009). Trading in Tv Entertainment: an Analysis. En Moran, A. (ed.). Tv formats worldwide. Localizing Global Programs. Bristol: Intelect.

Moran, A. (ed.) (2009 a). New Flows in Global TV. Bristol: Intelect.

Moran, A. (ed.) (2009 b). TV Formats Worldwide. Localizing Global Programs. Bristol: Intelect.

Oren, T. (2012). Reiterational Texts and Global Imagination. Television Strikes Back. En Oren, T. y Shahaf, S. (eds.). Global Television Formats: Understanding Television Across Borders (pp. 366-381). New York and London: Routledge.

Oren, T. y Shahaf, S. (2012). Introduction: Television Formats- A Global Framework for TV Studies. En Oren, T. y Shahaf, S. (eds.). Global Television Formats: Understanding Television Across Borders (pp. 1-20). New York and London: Routledge.

Oren, T. y Shahaf, S. (eds.) (2012). Global Television Formats: Understanding Television Across Borders. New York and London: Routledge.

Quevedo, L. (2006). La industria televisiva argentina. Identificación y caracterización de la producción televisiva argentina. Identificación 
y análisis del mercado de exportación de la industria televisiva argentina. Informe para el proyecto "Las Industrias Culturales Argentinas y su inserción en el Comercio Internacional”, Iesalc-Unesco.

Rohn, U. (2004). Media Companies and their Strategies in Foreign Television Markets. Arbeispapiere des Instituts für Rundfun kökonomiean der Universität. Recuperado de: http://www.rundfunk-institut.uni-koeln.de/institut/pdfs/18704.pdf.

Rohn, U. (2010). Cultural Barriers to the Success of Foreign Media Content. Western Media in China, India and Japan. Frankfurt: Peter Lang.

Roncagliolo, R. (1995). Trade integration and communication networks in Latin America. Canadian Journal of Communications, 20 (3), 335-342.

Saló, G. (2003). ¿Qué es eso del formato? Cómo nace y se desarrolla un programa de televisión. Barcelona: Gedisa.

Straubhaar, J. (2012). Telenovelas in Brazil. From Travelling Scripts to a Genre and Proto-Format both National and Transnational. En Oren, T. y Shahaf, S. (eds.). Global Television Formats: Understanding Television Across Borders (pp. 148-177). New York and London: Routledge.

The Wit (2014). Success Stories arround the World. The WIT Guide to Scripted Formats 2014. The Wit.

Waisbord, S. y Jalfin, S. (2009). Imagining the national: gatekeepers and the adaptation of global franchises in Argentina. En Moran, A. (ed.). TV Formats Worldwide. Localizing Global Programs (pp. 5574). Bristol: Intelect. 


\section{Listado de entrevistas en profundidad}

Antonini, Elena (28 de mayo de 2012). Encargada de distribución para Latinoamérica y Europa de Dori Media Group.

Badía, Marisa (25 de septiembre de 2012). Gerente de Señales Interior e Internacional de Telefe S.A.

Barberian, Virginia (14 de marzo de 2014). Director de Negocios Internacionales de Mediabiz.

Belón, Hernán (28 de mayo de 2012). Socio y director de Bestiana Films.

Borensztein, Guillermo (18 de junio de 2014). Gerente de Co-producciones y desarrollo de nuevos negocios de Telefe S.A.

Castany, Javier (8 de agosto de 2013). Gerente de Producción Ejecutiva de The Walt Disney Company Latin America.

Chamorro Magide, Leandro (16 de septiembre de 2013). Abogado. Biset $\&$ Chamorro Magide.

Cilley, Gonzalo (8 de marzo de 2013). Socio de Resonant TV.

Di Guglielmo, Hugo (1 de junio de 2012). CEO de HD International Media Consulting.

Ferrara, Fabricio (28 de mayo de 2013). Director de Negocios Internacionales de Prensario Internacional.

Geneste, Patty (14 de marzo de 2014). Directora de FRAPA (Format Recognition And Protection Association). CEO de Absolutely Independent.

González, Víctor (22 de octubre de 2013). Actualmente: socio de RGB Entertainment. 
Ipolitti, Claudio (18 de junio de 2014). Director de Negocios Internacionales y TV Paga de Telefe S.A.

Kirzner, Damián (29 de mayo de 2012). Productor General y director de Contenidos en New Sock.

Kon, Mariano (28 de mayo de 2012). Gerente General de A\&E Latin America.

Lagomarsino, Álex (11 de junio de 2012). CEO de MediaBiz.

Martínez Kolodziej, Juan Pablo (23 de julio de 2014). Director de Rodeo Films.

Muszalski, Pablo (16 de julio de 2012). Director de Win TV.

Otaola, Daniel (28 de mayo de 2014). Gerente de Distribución de Contenidos de Telefe S.A. (2013-presente).

Parra, Alejandro (14 de septiembre de 2012). CEO de Noujau International Consulting. Anteriormente: Director de Telefe Internacional (2000-2010).

Peretti, Agustina (12 de noviembre de 2012). Coordinadora de Promoción de Bienes Culturales de la Fundación Exportar.

Smirnoff, Miguel (17 de agosto de 2012). CEO de Editorial Prensario.

Smirnoff, Nicolás (14 de marzo de 2013). Director Prensario Internacional.

Tevah, Víctor (31 de agosto de 2012). Director de Nuevos Proyectos y Área Institucional de Pol-ka.

Varela, Fernando (1 de julio de 2014). Director de Asuntos Legales de Telefe (2001-presente).

Vibes, Federico (3 de agosto de 2012). Socio de Alesina y Asociados. Actualmente: Principal Counsel at ESPN. 
Vibes, Sebastián (23 de julio de 2013). Director de Desarrollo de Negocios en Televisa Internacional.

Waehner, Juan (1 de julio de 2014). CEO \& Presidente de Telefe S.A.

Wasserman, Michelle (1 de abril de 2012). Gerente de Negocios Internacionales y Desarrollo de Contenidos en Endemol Argentina. Anteriormente: gerente de negocios internacionales de Telefe Internacional (2007-2012).

Zilberman, Javier (14 de marzo de 2013). Productor. Desarrollo e investigación de formatos y venta internacional. Ideas del Sur. 